\title{
Classification of Pericarpium Citri Reticulatae of Different Ages by Using a Voltammetric Electronic Tongue System
}

\author{
Qingrui Shi ${ }^{1}$, Tingting Guo ${ }^{1}$, Tingjia Yin ${ }^{1}$, Zhiqiang Wang $^{1 *}$, Caihong Li $^{1}$, Xia Sun ${ }^{2}$, Yemin Guo ${ }^{2}$, \\ Wenhao Yuan ${ }^{l}$ \\ ${ }^{1}$ College of Computer Science and Technology, Shandong University of Technology, Zibo 255049 \\ P.R. China \\ ${ }^{2}$ College of agricultural engineering and food science, Shandong University of Technology, Zibo \\ 255049 P.R. China \\ *E-mail: $\underline{\text { wzq@sdut.edu.cn }}$
}

doi: $10.20964 / 2018.12 .45$

Received: 30 March 2018 / Accepted: 5 October 2018 / Published: 5 November 2018

\begin{abstract}
A portable voltammetric electronic tongue (VE-tongue) system was developed and used to classify pericarpium citri reticulatae (PCR), a traditional Chinese herbal medicine, on the basis of its age for authentication. An array of sensors with eight working electrodes (glass carbon, nickel, titanium, palladium, platinum, wolfram, gold and silver), a counter electrode and a reference electrode were used for signal collection. The feature data was further extracted from the raw signals by discrete wavelet transform (DWT). Seven linear and nonlinear classification methods, namely, principal component analysis (PCA), cluster analysis (CA), linear discriminant analysis (LDA), back-propagation neural network (BPNN), extreme learning machine (ELM), random forest (RF) and support vector machine (SVM), were compared for developing the discrimination model. The experimental results showed that the ELM model, in which the discrimination rates were $100 \%$ and $95 \%$ in the training and testing set, respectively, exhibited superior performance compared to the other models. The final results suggested that the VE-tongue system with the DWT-ELM classification method could be used to effectively identify PCR of various ages.
\end{abstract}

Keywords: Pericarpium Citri Reticulatae; Voltammetric electronic tongue; Discrete wavelet transform; Multivariate analysis

\section{FULL TEXT}

(C) 2018 The Authors. Published by ESG (www.electrochemsci.org). This article is an open access article distributed under the terms and conditions of the Creative Commons Attribution license (http://creativecommons.org/licenses/by/4.0/). 